\title{
Acquisition of meaning through context
}

\author{
Katherine E. Baker and Theo B. Sonderegger \\ UNIVERSITY OF NEBRASKA
}

\begin{abstract}
Ss, who were shown sentences with nonsense syllables replacing certain words, demonstrated: (a) enhanced syllable recognition, (b) interference in learning incorrectly paired syllables and replaced words, but (c) facilitation for correct pairings and (d) for pairings with associates of replaced words. Findings support the proposition that nonsense materials embedded in contexts with meaning acquire meaning.
\end{abstract}

\section{Problem}

The usual procedure for making nonsense stimuli acquire meaning is paired-associate learning with words with denotative or connotative meaning. This study investigated the hypothesis that an alternative procedure, embedding nonsense syllables in a context with meaning, also results in acquisition of meaning. Thus, in "For breakfast John ate ham and cef," cef should tend to evoke "eggs" and the associates of "eggs."

Previous studies (Werner \& Kaplan, 1950; Brown \& Berko, 1960) employing similar procedures showed Ss could replace such nonsense words with appropriate meaningful words on the basis of context, but they did not demonstrate that nonsense words themselves acquired specific differentiated meaningful response tendencies. In theory, covert responses of the missing word contiguous with presentation of the nonsense word should result in associations between the two words.

Specific hypotheses were: 1 . Nonsense materials embedded in contexts with meaning should gain meaning and thus be learned more easily than as meaningless syllables in contexts without meaning. 2. Subsequent paired-associate learning with the same nonsense syllables as stimuli should reflect associations acquired by exposure within contexts with meaning; (a) pairs relating syllables with words they replaced should show facilitated learning, (b) pairs relating syllables with words replaced by other syllables should show interference in learning. 3. Subsequent paired-associate learning relating the same nonsense syllables as stimuli and words meaningfully related to replaced words should show facilitated learning through mediated generalization.

Hypotheses 1 and 2 were tested in one experiment and a second experiment was run to test hypothesis 3 since increased discriminability of stimuli and/or specific response learning could account for results of hypotheses 1 and 2 while mediated generalization predicted in hypothesis 3 requires acquisition of meaning.

\section{Method}

In Experiment I, 66 experimental and 66 control college Ss were shown nine sentences, once each and one at a time, by means of an opaque projector in small group sessions. Experimental Ss had sentences like the one cited above pretested for agreement on the identity of missing words replaced by zero association nonsense syllables (Glaze, 1928). Control Ss had the same words but in scrambled order to supply a comparable context but without meaning, e.g., "breakfast ham for cef John and ate." All Ss read sentences to themselves along with $\mathrm{E}$ who read the words aloud slowly, presumably in a study of the effectiveness of different rates of reading. Following exposure to sentences, Ss were given a printed list containing the nine syllables from the sentences mixed with 11 other syllables and were asked to encircle those they had seen in the sentences.

Next, subgroups of $22 \mathrm{Ss}$ each were given one of three lists of nine paired-associates presented stimulus alone and then stimulus and response members together at a rate of $2 \mathrm{sec}$. per item. List $\mathrm{F}$ had the previously exposed syllables paired with their proper replaced words in sentences. List I paired these syllables with the same response words but in random order. List $\mathrm{N}$ paired the syllables with unrelated, "neutral" words of the same length as proper words in List F. Immediately after the last pair in whichever list was used, printed sheets containing the nonsense stimuli were passed out and Ss were given $2 \mathrm{~min}$. in which to write down the correct paired response words.

In Experiment II, 21 control and 18 experimental Ss went through the same procedures as in Experiment I with the exception that paired-associate learning was with List $G$ which consisted of the same nonsense syllables paired with words related in meaning to their proper words in List F. Words meaningfully related to replaced words were chosen from the most frequent associations a different group of $35 \mathrm{Ss}$ gave to the key words missing from sentences.

\section{Results}

Hypothesis 1, concerning recognition of syllables (Table 1A), was confirmed in both experiments with an F of 4.04 "between groups" in Experiment I and $t=2.47$ in Experiment II. Both differences were significant beyond the 5\% level. "Between subgroups" $\mathrm{F}$ and "subgroups by groups interaction" F were insignificant in Experiment I.

Hypothesis 2 was tested and supported with recall scores from Experiment I in Table IB which shows the predicted order of amount of learning, $\mathrm{F}>\mathrm{N}>\mathrm{I}$, for experimental Ss. The "lists by groups" F of 9.54 was significant beyond the $1 \%$ level, while "between lists" and "between groups" F's were not significant. The insignificant F's were to be expected since List I and List F differences were in opposite directions for experimental and control Ss and since differences between lists 
Table 1. Mean Learning Scores

A. Mean Number of Correct Recognitions

\begin{tabular}{cccc}
\multicolumn{1}{c}{ Experiment I } & Experimental & Control \\
& I & 6.50 & 5.86 \\
Subgroups & N & 6.05 & 6.00 \\
& F & 6.30 & 5.27 \\
Experiment II & 7.11 & 5.86
\end{tabular}

B. Mean Number of Correct Recalls

\begin{tabular}{lccc}
\multicolumn{2}{c}{} & \multicolumn{3}{c}{ Groups } \\
\multirow{2}{*}{ Experiment I } & Experimental & Control \\
& I & 3.23 & 4.86 \\
& $\mathrm{~N}$ & 3.59 & 3.64 \\
& $\mathrm{~F}$ & 5.45 & 3.59
\end{tabular}

\begin{tabular}{llll}
\multicolumn{2}{l}{ Experiment II } & & \\
Parts of & H & 2.83 & 2.09 \\
List G & L & 1.72 & 1.95
\end{tabular}

for control Ss (likely revealing inherent difficulty differences) were counteracted by treatments.

Hypothesis 3 was tested with recall scores on List G in Experiment II. On the total List G, experimental Ss were superior to control Ss $(M=5.44$ and 5.19 respectively), but, in fact, List $G$ was not uniform in strength of association between words and their key words. Some response items were associates given by more than $50 \%$ of the 35 preliminary Ss, while others were mentioned by fewer than $50 \%$, the most frequent associates having had to be eliminated because they also appeared in the sentences to be used. Therefore, a more informative comparison was made between recall scores for parts of List $\mathrm{G}$ shown in Table $1 \mathrm{~B}$, where $\mathrm{H}$ designates the four items ranking highest with respect to frequency of occurrence of the word chosen among preliminary Ss' associates and $\mathrm{L}$ designates the four items with the lowest frequencies. That is, $\mathrm{H}$ and $\mathrm{L}$ parts of the list represent high and low degrees to which response words were related to the replaced words in sentences.

As expected, degree of relatedness was a highly significant variable $(t=9.96)$ for experimental Ss and not significant for control Ss. Further, experimental Ss learned significantly more pairs than control Ss when response words were commonly associated with replaced words ( $t=1.77, P=.05$, one-tailed test) while there was no difference between groups on low frequency associates. Therefore, hypothesis 3 was supported with additional evidence for extent of mediated generalization effects being a function of degree of relatedness between word responses and the key words to which they were associates.

\section{Diseussion}

The results provide strong support for the hypothesis that meaning may be imparted to nonsense material merely by a context with meaning. Recognition scores do not supply unequivocal evidence because they may reflect acquired meaning and/or greater discriminability of syllables. However, subgroups of experimental Ss all with equivalent recognition scores, and possible advantages of stimulus predifferentiation, show differences in subsequent paired-associate learning depending upon whether there is positive or negative transfer from learning during exposure to sentences. That the learning is not simply a case of bare associated responses without any meaning is attested to by the demonstrated mediated generalization to related words.

The intent of the study was to identify a source of acquired meaning rather than to specify mechanisms for learning such associations. Nevertheless, it is feasible to speculate that contexts with meaning such as those used probably represent well practiced chains of responses which provide for there being covert responses where words are missing. Syllables present contiguously with such covert responses may be expected to become associated with those responses and with other responses in turn associated with them. Providing it is accepted that associations like "ham $\rightarrow$ eggs" can be supplied by a context, the present experiments can be conceptualized according to the paradigms of mediated association of Jenkins, Russell and their colleagues (Jenkins, 1963). Experiment I exemplifies facilitation expected from their response equivalence design, while Experiment II demonstrates facilitation predictable from a combination of response equivalence and simple chaining designs. Thus, the demonstrated role of context in acquisition of meaning does not appear to call for any new learning mechanisms. The major implication of the study is that contextual factors need to be recognized as having potent and rapid effects upon behavior. A single exposure to a syllable in a context with meaning affected the very first paired-associate learning trial even if responses were only related words.

\section{References}

BROWN, R., \& BERKO, JEAN. Word association and the acquisition of grammer. Child Developm., 1960, 31, 1-14.

GLAZE, J. A. The association value of nonsense syllables. J. genet. Psychol., 1928, 35, 255-267.

JENKINS, J. J. Mediated associations: paradigms and situations. In C. N. Cofer \& Barbara S. Musgrave (Eds.), Verbal behavior and learning: problems and processes. New York: McGraw-Hill, 1963. Pp. 210-245.

WERNER, H., \& KAPLAN, EDITH. The acquisition of word meanings: a developmental study. Child Develpm. Pub. Monogr., 1950, 15, No. 51, No. 1. 